Part of Journal of Research of the National Bureau of Standards, Volume 30, January 1943

\title{
AN IMPROVED ELECTRODE HOLDER FOR SPECTROGRAPHIC ANALYSIS*
}

\author{
By Bourdon F. Scribner and Charles H. Corliss
}

\section{ABSTRACT}

An electrode holder has been constructed for improving the speed and ease of operations in miscellaneous spectrographic testing. Advantages afforded by the new holder include: (1) application to a variety of electrode sizes and excitation conditions, (2) positive and precise adjustment and motion of the parts, (3) watercooling of the electrode clamps, and (4) a housing for protection against light, fumes, and electric shock. The construction of the holder and its application to excitation problems with the high-voltage spark, the a-c arc, and the d-c arc are described.

\section{CONTENTS}

I. Introduction

II. Design and construction

1. General features

2. Electrode clamps

3. Water-cooling system

4. Housing

III. Applications of the holder

IV. References..... 45

\section{INTRODUCTION}

Many of the parts of the spectrographic assembly have undergone marked improvement during recent years, but relatively little attention has been given to the design of the electrode bolder, except for certain special applications. In addition to the common models based on the simple Gramont design, holders have been devised for a-c arc excitation of rods $[1,2]^{1}$ or sheet metal [3], and for rapid change of spark electrode rods $[4,5]$ or metal plates [6]. A universal holder has been described [7] that provides a variety of electrode movements and adjustments.

For application in miscellaneous analyses, however, difficulty may be encountered when changes must be made quickly to handle electrodes of different sizes and shapes and from one extreme of excitation conditions to another. To meet these demands, a holder has been constructed incorporating the following features: (1) ease and speed of operation over the varied conditions encountered, (2) precise adjustment and motion of parts to prevent errors caused by poor alinement

*Presented at the Tenth Annual Conference on Applied Spectroscopy (at MIT) on July 22, 1942.

1 Figures in brackets indicate the literature references at the end of this paper. 
of electrodes, (3) water-cooling of electrode clamps to remove excessive heat, thus preventing deterioration of the parts and, at the same time, cooling the electrodes when necessary, (4) provision for adjustment of distance of holder from the spectrograph slit by an optical bench to permit variation in illuminating conditions, and (5) a hood for protection against light, fumes, and electric shock.

The design of the holder to be described is based on the suggestions of several operators and incorporates improvements found advisable by tests on a preliminary model.

\section{DESIGN AND CONSTRUCTION}

\section{GENERAL FEATURES}

A view of the holder, with the housing opened to expose the parts, is given in figure 2. Details of its design and construction are shown in the assembly drawing reproduced in figure 1 . The holder consists of a heavy steel base, 1, standing on three legs (two V's and a flat) that rest on the optical bench. The base is locked to the bench by a claw, 2, and is fitted with a slide that is moved transversely by control, 3. The slide carries two steel upright rods, 4, that guide the electrode supports, 5. Each support consists of an $\mathrm{H}$-shaped brass yoke, constructed from tubing and block brass, with a forward extension upon which is bolted a Bakelite plate, 6, carrying the electrode clamp, 7. The uprights, 4, are braced at the upper ends by a plate, 8 , on which is mounted the upper control assembly, 9. This control provides vertical movement of the upper electrode support through miter gears and a screw, 10, threaded into the support. The screw chosen for this holder has a pitch of $1.5 \mathrm{~mm}$. Precise setting of small electrode distances is provided by a drum, 11, mounted on the screw and graduated with 15 divisions, each division corresponding to a $0.1-\mathrm{mm}$ travel of the electrode support. Independent motion of the lower electrode support is provided by a control, 12, with gears and a screw, 13, similar to the upper electrode assembly. The exposed metal parts, except the electrode clamps, are nickel-plated to resist corrosion.

\section{ELECTRODE CLAMPS}

Enlarged views of one of the electrode clamps are shown in section $D$ of figure 1 . The clamp jaws are made of two pieces of copper, one forming the movable jaw, 14, and the other the fixed jaw, 15. The motion of the jaw, 14, is precisely limited and controlled by a slide and ways on each side of the clamp. A quarter-turn of the knob, 18, provides the necessary opening and closing of the jaw (1/16 in.) through an eccentric shaft, 17 . A spring, 20, provides a fixed tension on the jaw for clamping the electrodes. The clamp assembly extends through the opening between the holder uprights and terminates in a binding post for connection to electric power. The back cover, 22, of the eccentric and spring box is Bakelite, and the face cover, 23, exposed to the source is made of brass.

The electrode jaws are drilled to accommodate a variety of electrode diameters for the lower and upper clamps, respectively, as follows: $1 / 2$ in. vs $1 / 2$ in., $1 / 4$ in. vs $1 / 8$ in., $1 / 4$ in. vs $1 / 4$ in., and $7 / 32$ in. vs $7 / 32$ in. Slight variations from the standard electrode sizes cause no difficulty in clamping if the holes are made slightly oversize. Split cop- 

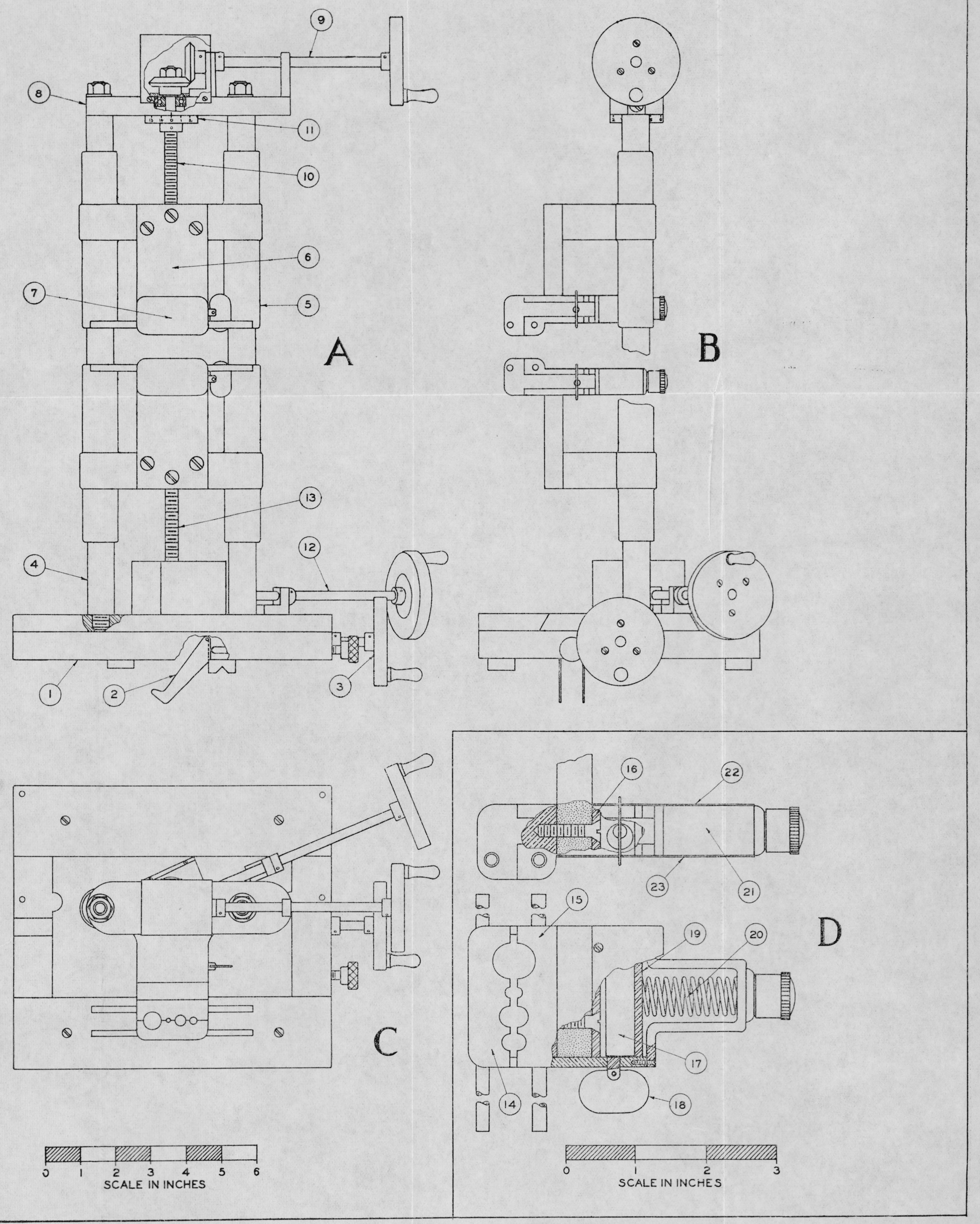


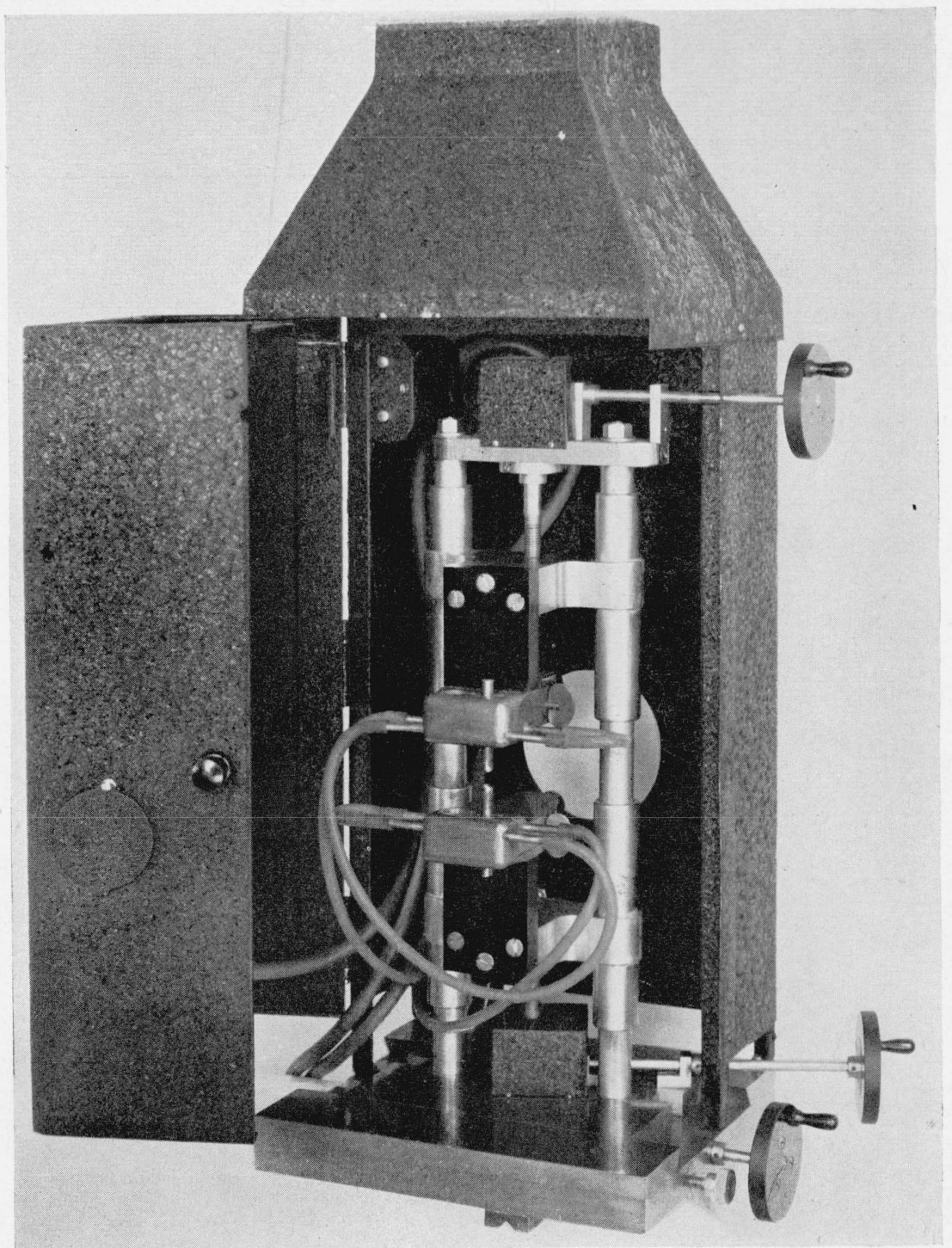

Figure 2.-Improved electrode holder. 


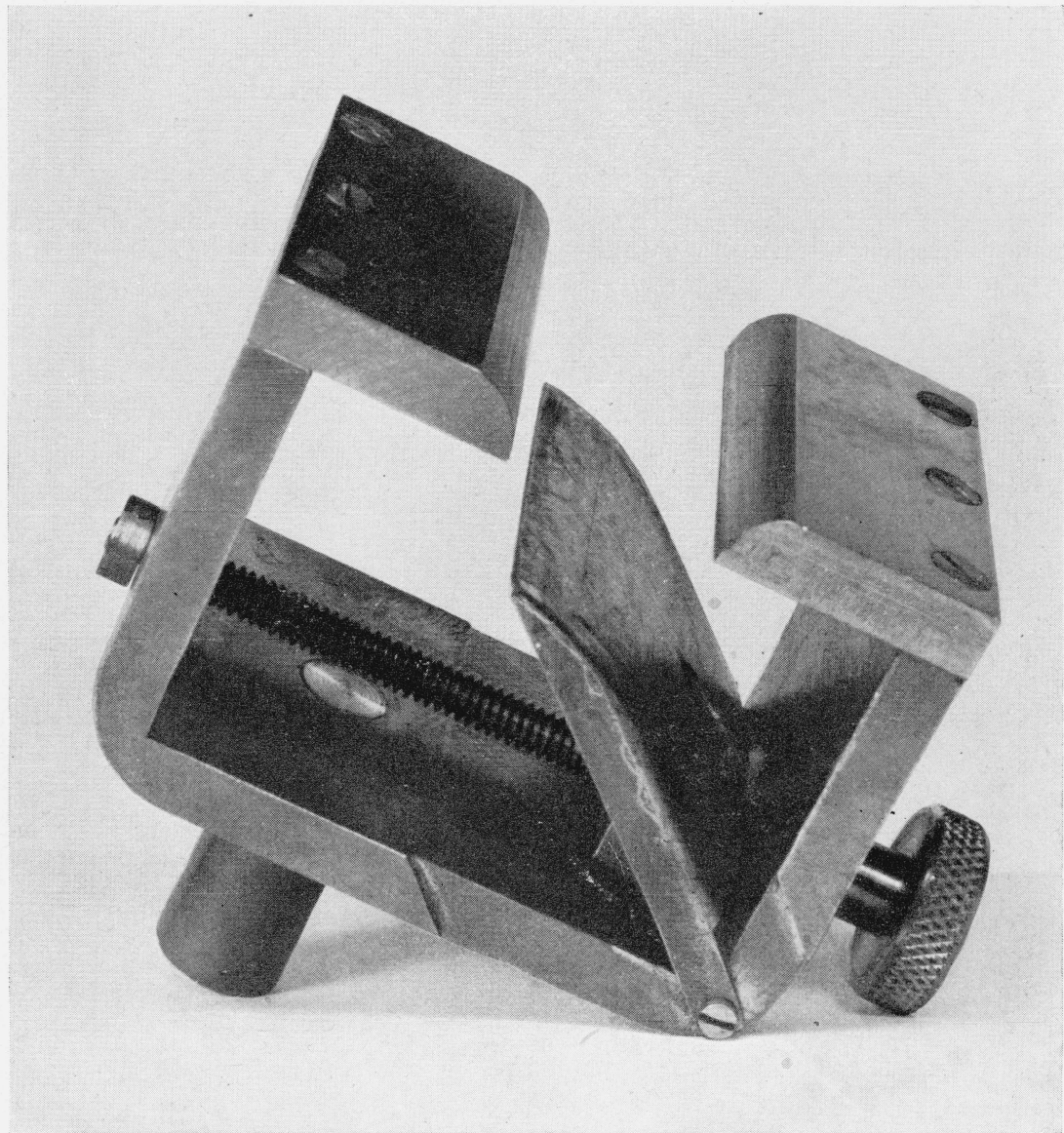

Figure 3.-Accessory clamp for holding disk and sheet samples. 
per bushings, machined to fit the jaws, permit other electrode combinations. The jaws of the clamp, once accurately alined, have maintained alinement during continuous use. All corners and edges of the jaws are rounded and the parts streamlined to facilitate cleaning. Copper tubes inserted through the jaws serve for water circulation. Finally, an accessory clamp shown in figure 3, which accommodates flat bar, disk, or sheet samples, may be quickly locked into either the upper or lower electrode clamp.

\section{WATER-COOLING SYSTEM}

The electrode jaws are cooled by water circulated from a reservoir (5-gal bottle) by means of a small rotary pump, the water flowing successively through the four jaws of the clamps before returning to the reservoir. Connections in the water system are made by gum-rubber tubing and glass. This, combined with the use of distilled water in the system, is effective in preventing the leakage of high-voltage (35,000-v peak) power. The closed water system, always at room temperature or slightly higher, ensures freedom from condensation of moisture on the holder when not in use. As a convenience in operation, the pump motor is connected to power through a master switch which closes electric circuits to the spectrograph controls, a rotating sector, and a ventilator. Under these conditions the water-cooling system requires little attention from the operator.

\section{HOUSING}

The housing of the holder is built with a frame of $1 / 2$-in. angle iron and a covering of galvanized sheet iron, and is finished in black crackle enamel. The forward half of the housing, which is supported on hinges at the side, forms a door to provide access to the holder. When the door is opened, a switch and safety circuit shut off the power circuit to the electrode clamps and a second switch closes a circuit to a lamp mounted in the corner of the door. The door opens to a stop so arranged that the lamp is placed in a position to project an image of the electrodes to a screen on the optical path for adjustment of the electrode positions. The lamp serves also to illuminate the interior of the housing.

Openings are cut in the front and back of the housing for the passage of light along the optical path. Ventilation is provided by a hood at the top of the housing, connected through a flexible tube to a ventilating system. A small upward current of air is sufficient; excessive air currents will make an arc discharge unsteady or even extinguish it. The housing is grounded through the base of the holder and the optical bench.

\section{APPLICATIONS OF THE HOLDER}

The holder has been employed for spark excitation with the various electrode combinations employed in the Bureau's spectroscopic laboratory. These include the spark between metal rods of sizes from $1 / 8$ in. to $1 / 2$ in. in diameter, between $1 / 8$-in. graphite rod and flat metal specimens, and between pressed pellets $1 / 4$ in. in diameter and $1 / 4$ in. to $1 / 2$ in. long. Cooling of the spark electrodes is useful when small electrodes or sheet-metal specimens are sparked. Sheet-metal samples may be mounted either vertically in the slot between the jaws 
of the lower clamp or held horizontally in the accessory holder (fig. 3), with a supporting block of copper for rapid heat conduction. The sample in this case is opposed by a counter electrode of graphite. The holder has been found advantageous in establishing a reproducible condition of heat conduction from the electrodes. With holders previously used in this laboratory, considerable variation in conduction has been encountered, especially after deterioration of the holders in use.

Excitation by the a-c arc generally requires close control of alinement and gap distance of electrodes. In order to set the electrodes accurately for small gaps, the upper electrode is lowered almost in contact with the lower electrode, the clamp is opened slightly, permitting the upper electrode to drop into contact with the lower, the clamp is then released to grip the electrode, and, finally, the electrode is raised the required distance, which is measured on the graduated drum (fig. 1, part 11). The holder was found satisfactory not only in meeting the requirement of accuracy in the settings, but also in providing the advantage of cooling the electrodes. Small pellets or 1/8-in.-rod samples may be arced with little danger of overheating.
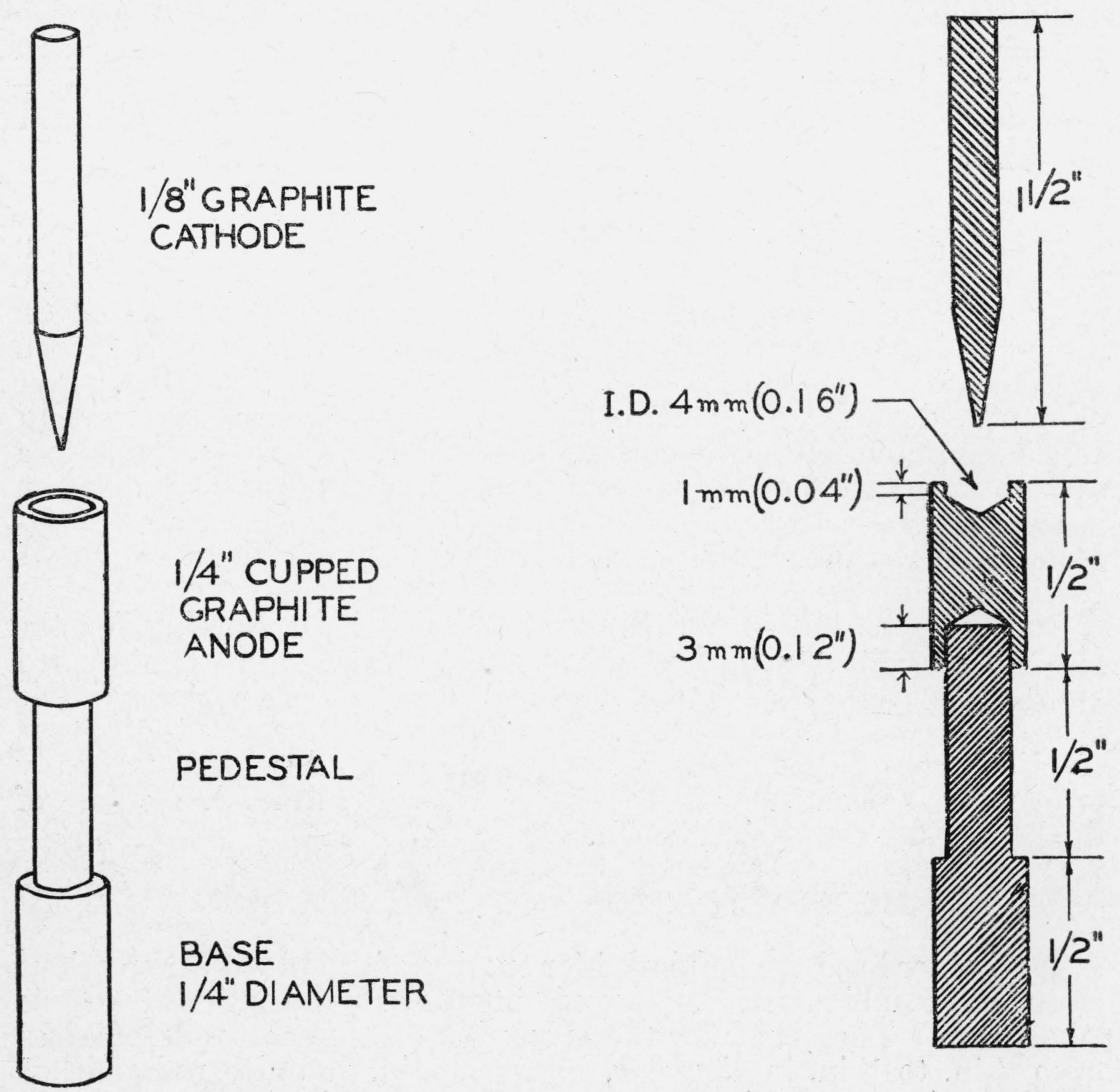

FIGURE 4.-Electrode assembly for direct-current arc excitation. 
Direct-current arc excitation results both in radiation of heat and in evolution of hot, corrosive vapors. The design of the electrode clamps and efficient water cooling have proved effective in meeting these conditions for rapid and continuous use of the holder. The cooling of electrodes of various metals in the form of compressed pellets permits use of currents as high as $10 \mathrm{amp}$ without danger of melting the electrode except at the end. However, if heat is required in the electrode, as in volatilizing samples from a hot graphite anode, unsatisfactory results may be observed with the customary electrode shapes. As a result of the high cooling efficiency of the holder, the electrodes are attacked only on the extreme ends with currents as high as $15 \mathrm{amp}$. To overcome this difficulty, the d-c arc electrode assembly was modified to provide an electrode combination that has proved adaptable to a number of applications of the d-c arc, both for the water-cooled holder and common clip holders. The assembly, with dimensions shown in figure 4 , consists of a $1 / 8$-in. upper cathode tapered to a point and a lower anode combination in two partsfirst, a graphite base and pedestal supported in the electrode clamp; and, second, the electrode cap. The cap is drilled at both ends, the depth of the upper cup depending on the degree of heating required and the size of sample charge. By virtue of the low heat conductivity between the cap and the pedestal, the cap becomes very hot with only slight attack on the pedestal. This electrode combination gives reproducible excitation and requires a smaller quantity of graphite rod, which is a welcome economy when expensive high-purity electrodes must be used.

Experience with two of the improved electrode holders for numerous tests under various conditions shows that they have increased the speed of miscellaneous testing, provided more reproducible electrode orientation, and contributed to the safety and ease of operations.

The authors acknowledge the helpful suggestions of H. R. Mullin, of the Spectrographic Laboratory, and of Henry Philo, of the Bureau's Instrument Shop.

\section{REFERENCES}

[1] O. S. Duffendack and R. A. Wolfe, Ind. Eng. Chem., Anal. Ed. 10, 161 (1938).

[2] W. D. Owsley and R. C. MeReynolds, Rev. Sci. Instr. 13, 342 (1942).

[3] R. A. Sawyer and H. B. Vincent, J. Applied Phys. 11, 452 (1940).

[4] Carl Zeiss, German Patent 681,294, Aug. 31, 1939.

[5] H. B. Vincent and R. A. Sawyer, Proceedings Seventh Summer Conference on Spectroscopy and Its Applications, p. 16 (John Wiley \& Sons, New York, N. Y. $(1940)$.

[6] H. V. Churchill and J. R. Churchill, J. Opt. Soc. Am. 31, 611 (1941).

[7] A. Gatterer, Ric. Spettroscopiche 1, 55 (1939).

WASHINGTON, October 22, 1942. 